\title{
MAGNETIC FLOCCULATION OF HEMATITE MINERALS
}

\section{Y. SHAO, T.J. VEASEY ${ }^{\star}$ AND N.A. ROWSON}

School of Chemical Engineering, The University of Birmingham, Edgbaston, Birmingham B15 2TT, U.K.

${ }^{\star}$ Department of Chemical Engineering, National University of Science and Technology, P.O. Box 346, Bulawayo, Zimbabwe

(Received February 2, 1996, accepted March 19, 1996)

\begin{abstract}
The magnetic flocculation behaviour of two kinds of iron oxide mineral (hematite-1 and hematite-2) was studied by using a new experimental device, in which a stirred container was set in an applied magnetic field. Magnetic flocculation of both minerals occurred in very low applied magnetic fields. Hematite- 1 is a strongly paramagnetic mineral, the flocculation recovery improved with increased magnetic field strength and particle size. For hematite-2 the relationship between the flocculation recovery and magnetic field strength was more complex. It was also found that the method used conventionally to calculate the magnetic interaction between particles is not suitable for strongly paramagnetic minerals, such as hematite-1.
\end{abstract}

\section{INTRODUCTION}

In the mineral beneficiation of paramagnetic iron ores, wet high intensity magnetic separation and flotation are commonly used to separate weakly magnetic iron minerals from the unwanted gangue minerals $\left(\mathrm{SiO}_{2}\right.$, etc. $)$. It has been reported [1] that these separation processes are not effective on ultrafine [articles and are very sensitive to the quantity of ultrafine particles contained in the mineral pulp, because of their high specific surface and small mass. Thus, desliming is commonly used to reduce the adverse effect of ultrafine particles prior to flotation or magnetic separation. 
When finely disseminated iron ores are treated, conventional desliming of a fully dispersed pulp causes an excessive loss of valuable iron minerals to the tailings, leading to an increase in production costs and possible environmental problems. Therefore, the need for an improved method of treating fine minerals has been widely recognised, and a great number of studies on the recovery of fine valuable minerals have been reported [2].

Magnetic flocculation is potentially attractive process for treating such materials, in which the iron minerals selectively aggregate to become more readily treatable. Recent research [3] indicates that fine magnetite can induce the flocculation of hematite fines. Adding flocculant or long-chain surfactant to the pulp can increase the strength of aggregates formed, and wet high-gradient magnetic separation can effectively separate these aggregates from the dispersed gangue $[4,5]$. However, the added magnetite particles can cause blockages in the magnetic separator matrix.

Other researchers $[6,7]$ used an external magnetic field to induce the flocculation, but the properties of flocs have not been investigated. The separation of the flocs from the dispersed gangue minerals used settling methods which can often give poor separation efficiencies. In the present study, the magnetic flocculation of hematite minerals was studied in an magnetised gap with a stirrer. The strength of the flocs and the rate of flocculation were observed.

\section{EXPERIMENTAL PROCEDURE}

\section{Materials and Devices}

In this study, two kinds of hematite mineral with different magnetic susceptibility were chosen, e.g. hematite-1 and hematite-2. These minerals were, respectively, wet ground by using a laboratory steel-ball mill, and seized by a laboratory cyclosizer (Warman International (Pty) Ltd., Australia). Five size fractions of each mineral were collected as the test samples.

For the magnetic flocculation test, the traditional method is to put a settling tube into a magnetised gap, and then place the fully dispersed pulp into this gap and 
the the same time observe the formation of flocs or the change of particle settling rate. Using this kind of method, the flocs obtained are commonly loose and chain-like, the collision, which usually controls the occurrence and rate of flocculation, between magnetisable particles cannot be controlled.

In this study, a new experimental device for magnetic flocculation was designed, and is shown in Fig. 1. A laboratory wet high intensity magnetic separator (Type LHWL, made by boxmag-Rapid Ltd., England) was used to produce the background magnetic field for magnetic flocculation. The dimensions of gap between magnetic poles is $81 \times 32 \times 136 \mathrm{~mm}$. The maximum magnetic field strength is about 1.5 Tesla. The range of adjustable stirring speed is about 40 to $2000 \mathrm{rpm}$.

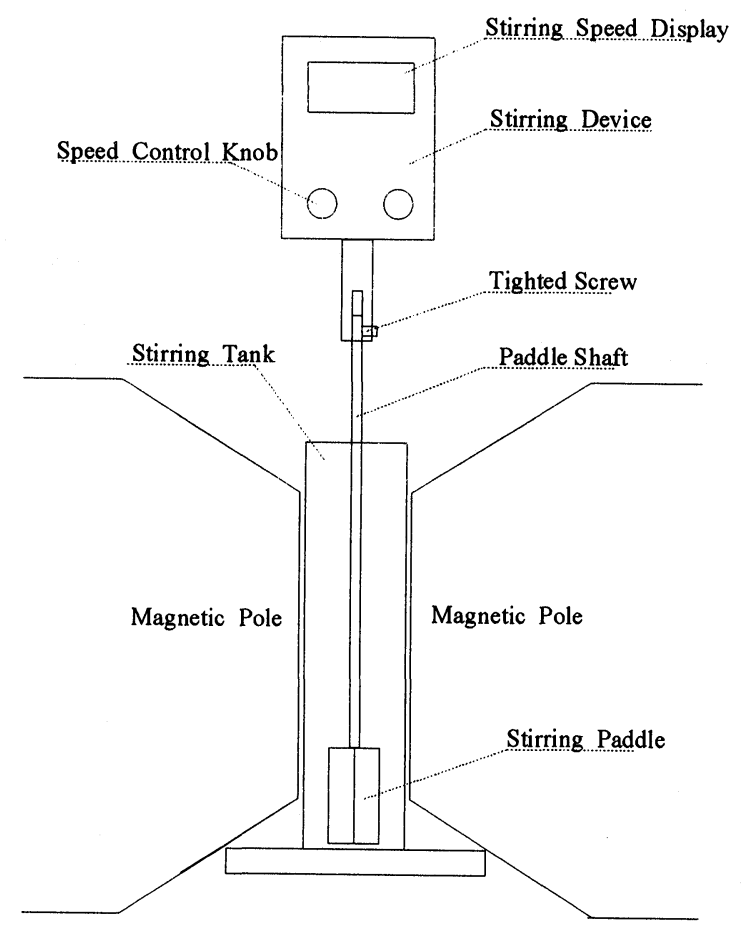

Fig. 1 Magnetic flocculation test equipment

Using this device, compact flocs can be obtained, which should be advantageous to the choice of downstream separation technique. The probability of collision between particles can be controlled by adjusting stirrer speed and time. 
Analytical grade sodium hydroxide and hydrochloric acid were used to adjust the pulp $\mathrm{pH}$ value. Deionised water was used throughout the study. A $\mathrm{pH}$ meter was used to measure the pulp $\mathrm{pH}$.

\section{Method}

A known weight of sample was wetted with deionised water to make pulp of about $2 \%$ solids by weight. The pulp was intensely stirred for about 8 hours to ensure that particles were fully dispersed, and the diluted to $200 \mathrm{~g} / \ell$. In each test, the diluted pulp $(60 \mathrm{ml})$ was placed into the settling tube, and the pulp $\mathrm{pH}$ was adjusted. The settling tube was placed into the gap between the magnetic poles, the stirring speed was adjusted, and then the magnetic field was switched on. After stirring for a given time, the stirrer was stopped, and the magnetic field was switched off at the same time.

After standing for a set settling time, the upper suspension in the tube was removed from the sediment which was considered to be the flocculated product. Both of the products were, respectively, filtered, dried and weighed. The efficiency of magnetic flocculation was determined by the weight percentage of flocculated part to the total weight of the two products.

The settling rate of particles was observed at the natural $\mathrm{pH}$. It was found that in the early period of settling, particles could move down at the free-falling rate. After settling for about 2.5 minutes, the particle settling became very slow. In order to better observe the magnetic flocculation of hematite, the settling time was determined when the weight percentage of settled material was equal to about $50 \%$.

\section{RESULTS}

Figure 2 shows the effect of pulp $\mathrm{pH}$ on the magnetic flocculation of hematite minerals. Without an applied magnetic field, in the range of $\mathrm{pH}=2$ to 10 , the sediment percentage of hematite-2 hardly changed with the pulp $\mathrm{pH}$, and for hematite-1, it seems that flocculation had occurred and the sediment percentage changed with the pulp $\mathrm{pH}$. 


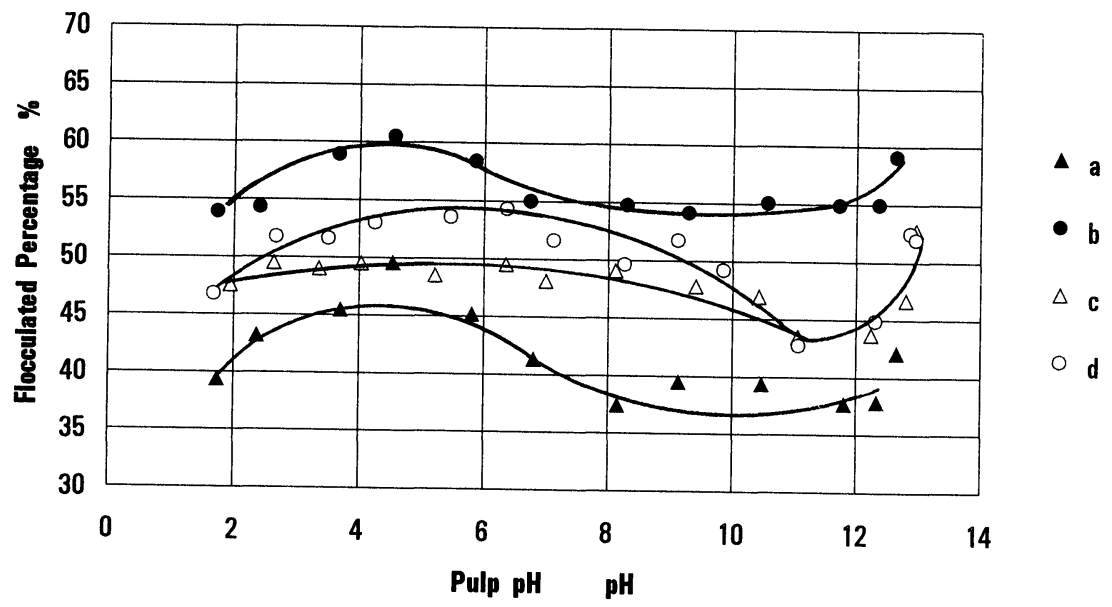

Fig. 2 The effect of pulp $\mathrm{pH}$ on the magnetic flocculation of hematite minerals. Stirring speed $500 \mathrm{rpm}$, stirring time 5 minutes; (a) and (b) for hematite-1, (c) and (d) for hematite-2; (a) and (c) without magnetic field, (b) and (d) in an applied magnetic field.

When an applied magnetic field was used, the sediment percentage of hematite-2 changed with the pulp $\mathrm{pH}$, and the flocculated percentage increased from $49 \%$ to $53 \%$ at $\mathrm{pH}=5.5$. The flocculated percentage value of hematite- 1 increased from $49 \%$ to $60 \%$ at $\mathrm{pH}=4.5$.

From Fig. 2, it can also be seen that after the pulp $\mathrm{pH}$ was raised above 12, the flocculated percentage of both minerals increased with pulp $\mathrm{pH}$, whether or not the magnetic field was used.

Figures 3 and 4 show the effect of applied magnetic field strength and particle size on the magnetic flocculation of hematite minerals. For hematite-1, the efficiency of magnetic flocculation increased with the increase in applied magnetic field and the particle size. When the field strength was $<0.1$ Tesla, coarser particles showed a more rapid increase in flocculation efficiency than finer particles. 


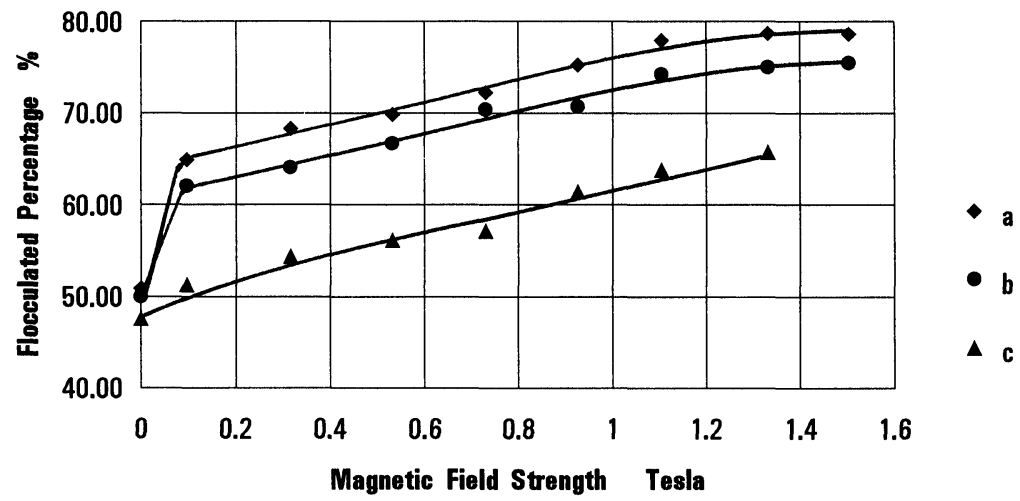

Fig. 3 The effect of applied magnetic field strength and particle size on the magnetic flocculation of hematite-1. Stirring speed $500 \mathrm{rpm}$, stirring time 30 minutes, pulp $\mathrm{pH}=4.5$. (a) $-23+15 \mu \mathrm{m}$, (b) $-15+11 \mu \mathrm{m}$, (c) $-11 \mu \mathrm{m}$.

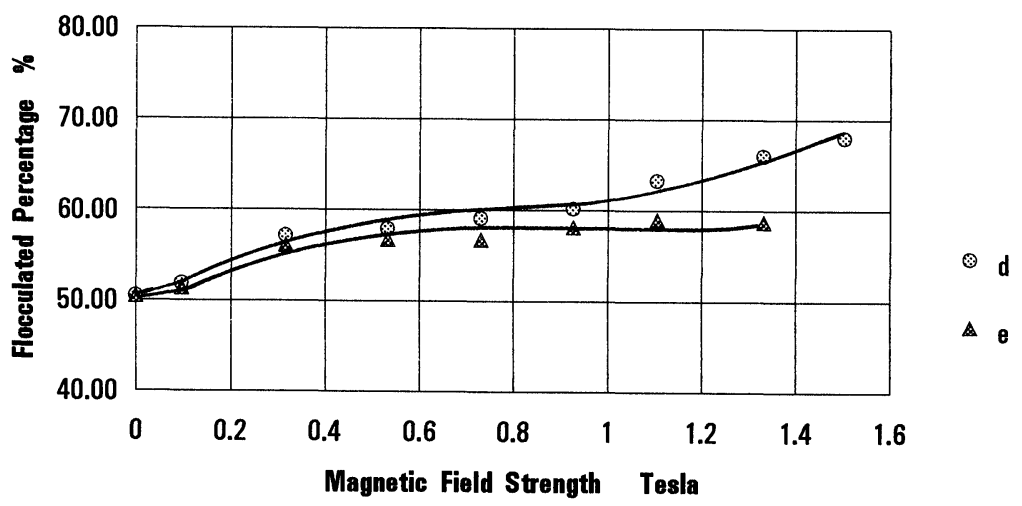

Fig. 4 The effect of applied magnetic field strength and particle size on the magnetic flocculation of hematite-2. Stirring speed $500 \mathrm{rpm}$, stirring time 30 minutes, pulp $\mathrm{pH}=\mathbf{5 . 5}$. (d) $-15+11 \mu \mathrm{m}$, (e) $-11 \mu \mathrm{m}$.

For hematite-2, the influence of magnetic field strength and particle size on magnetic flocculation was more complicated. The behaviour of coarser particles was similar to hematite-1 particles, but for $-11 \mu \mathrm{m}$ hematite-2 particles the efficiency of magnetic flocculation did not always increase with magnetic field strength. When the field strength was smaller than 0.1 Tesla, magnetic 
flocculation did not occur; when the field strength was in the range of 0.1 to 0.4 Tesla, the efficiency of magnetic flocculation increased with the field strength; and after the field strength was greater than 0.4 Tesla, the flocculation efficiency approached a maximum.

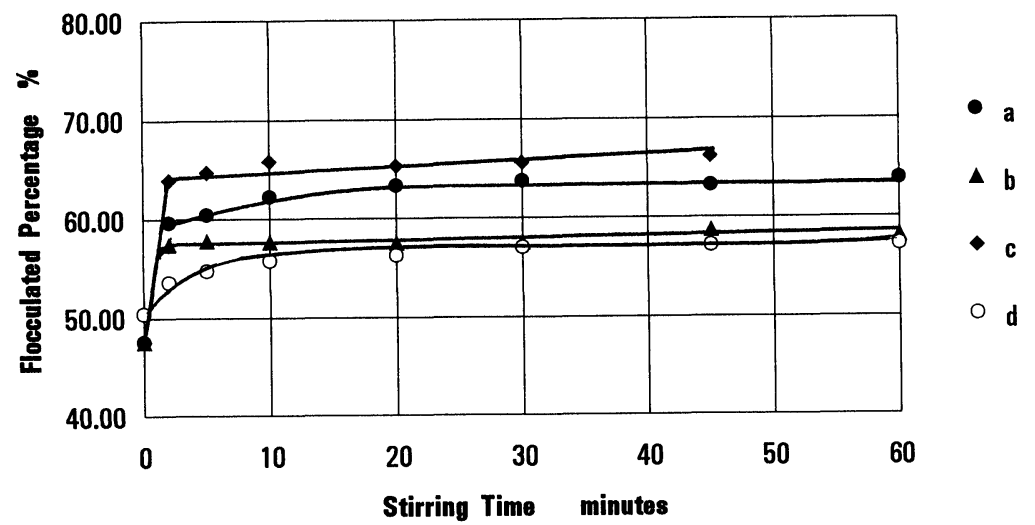

Fig. 5 The effect of stirring time on the magnetic flocculation of -11 $\mu \mathrm{m}$ hematite minerals. For hematite -1 , pulp $\mathrm{pH}=4.5$, for hematite-2 $\mathrm{ph}=5.5$. (a) hematite-1, stirring speed $500 \mathrm{rpm}$, magnetic field $1.024 \mathrm{~T}$; (b) hematite-1, stirring speed 700 rpm, magnetic field $1.024 \mathrm{~T}$; (c) hematite-1, stirring speed $500 \mathrm{rpm}$, magnetic field $1.332 \mathrm{~T}$; (d) hematite-2, stirring speed $500 \mathrm{rpm}$, magnetic field $1.024 \mathrm{~T}$.

Figure 5 shows the effect of the stirring time on the magnetic flocculation of -11 $\mu \mathrm{m}$ hematite minerals. It can be seen that the rate of magnetic flocculation was rapid at the beginning; after stirring for 2 minutes the flocculation efficiency increased slowly; and after stirring for 15 minutes the flocculation efficiency was up to a maximum.

Increasing the stirring speed could shorten the stirring time required to reach this maximum, but at the same time would cause a decrease in the flocculation efficiency. Increasing the magnetic field strength not only could shorten the stirring time required, but also could improve the flocculation efficiency.

Figure 6 shows the effect of stirring speed on the magnetic flocculation of hematite minerals. The flocculation efficiency decreased with increase of stirring speed. 
These results suggest that during magnetic flocculation, the stirring speed used should be as slow as possible.

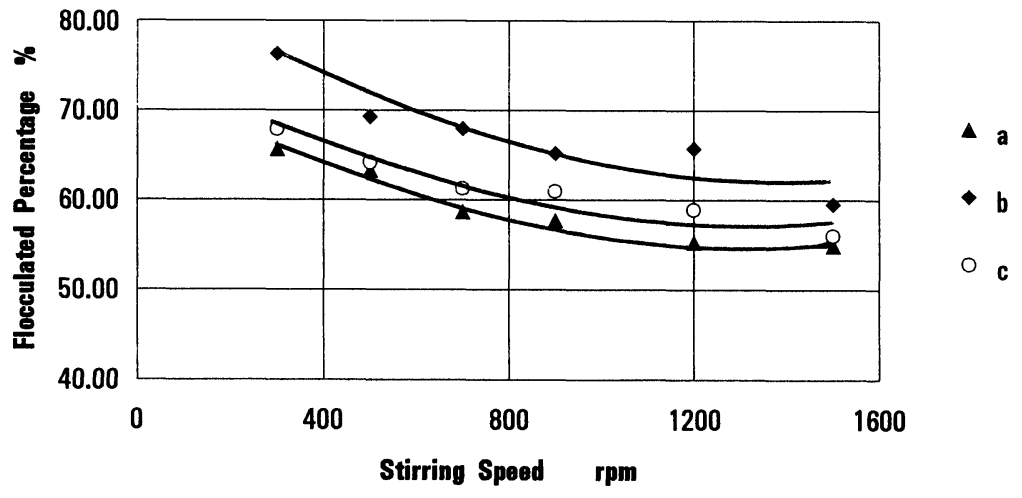

Fig. 6 The effect of stirring speed on the magnetic flocculation of hematite minerals. Stirring time 20 minutes, magnetic field strength 1.024 T. (a) $-11 \mu \mathrm{m}$ hematite -1 , pulp $\mathrm{ph}=4.5$; (b) $-15+11 \mu \mathrm{m}$ hematite-2, pulp $\mathrm{pH}=4.5$; (c) $-15+11 \mu \mathrm{m}$ hematite-2, pulp $\mathrm{pH}=5.5$.

Figure 7 shows the effect of pulp concentration on the magnetic flocculation of hematite-1 mineral. In the range of pulp concentration tested, no obvious influence was found.

\section{DISCUSSION}

According to the DLVO coagulation theory [8], the stability of a suspension of colloidal particles is largely governed by the interplay of repulsive and attractive forces between particles. The repulsive interaction $\left(V_{R}\right)$ between two colloidal particles can be expressed as:

$$
\mathrm{V}_{\mathrm{R}}=\mathrm{f}_{(\mathrm{a})^{\cdot} \cdot \mathrm{g}}\left(\kappa, \psi_{0}\right)
$$


where $a$ is the particle radius, $\psi_{0}$ is the surface potential of particles, $\kappa$ is the Debye-Huckel reciprocal length and is related to the ionic strength of the medium.

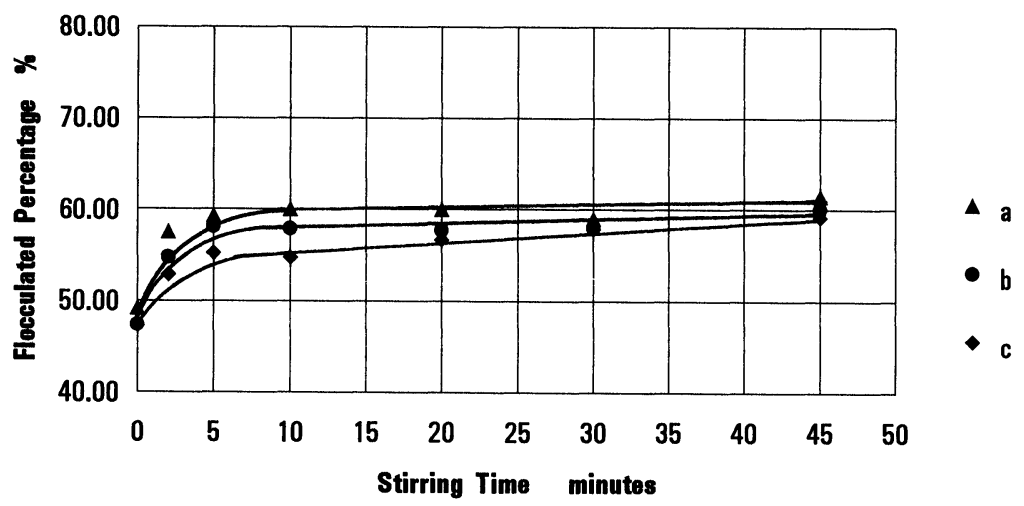

Fig. 7 The effect of pulp concentration on the magnetic flocculation of $-11 \mu \mathrm{m}$ hematite -1 mineral. Pulp $\mathrm{pH}=4.5$, stirring speed $500 \mathrm{rpm}$, magnetic field strength $1.024 \mathrm{~T}$. (a) $100 \mathrm{~g} / \ell ;$ (b) 300 $\mathrm{g} / \ell ;(\mathrm{c}) 400 \mathrm{~g} / \ell$.

The attractive interaction $\left(V_{A}\right)$ between two colloidal particles can be expressed as

$$
V_{A}=f_{(a, r, A)}
$$

where $r$ is the shortest distance of approach between the surfaces, $A$ is the Hamaker constant and is related to the dielectric properties of particles.

It can be seen from the above equations that for a given material, after the particle size is determined, the stability of a suspension will depend on the ionic strength of medium. When sufficient amount of electrolyte is added to a suspension, the repulsive interaction will decrease and coagulation between particles will occur. In this study, $\mathrm{NaOH}$ was employed as electrolyte, and when the pulp $\mathrm{pH}$ was greater than 12, a large addition of $\mathrm{NaOH}$ was required. Thus, the coagulation between particles occurred, and the sediment percentage increased. 
When a suspension of colloidal particles is placed in an applied magnetic field, there is, in addition to the forces considered above, an attractive magnetic force. The magnetic attraction $\left(V_{M}\right)$ is approximated by [9]:

$$
\mathrm{V}_{\mathrm{M}}=-\frac{32 \pi^{2} \mathrm{a}^{6} \chi^{2} \mathrm{~B}^{2}}{9 \mu_{0} \mathrm{r}^{3}}
$$

where $\chi$ is the volume magnetic susceptibility of particles, $B$ is the magnetic induction, and $\mu_{0}$ is the magnetic permeability of vacuum.

It can be found from this equation that for a given particle size, the magnetic attraction will increase with the magnetic susceptibility of particles and the applied magnetic field.

To better understand the effect of the magnetic attraction on the flocculation of hematite particles, the magnetic susceptibilities of two kinds of hematite minerals were measured and are shown in Figure 8.

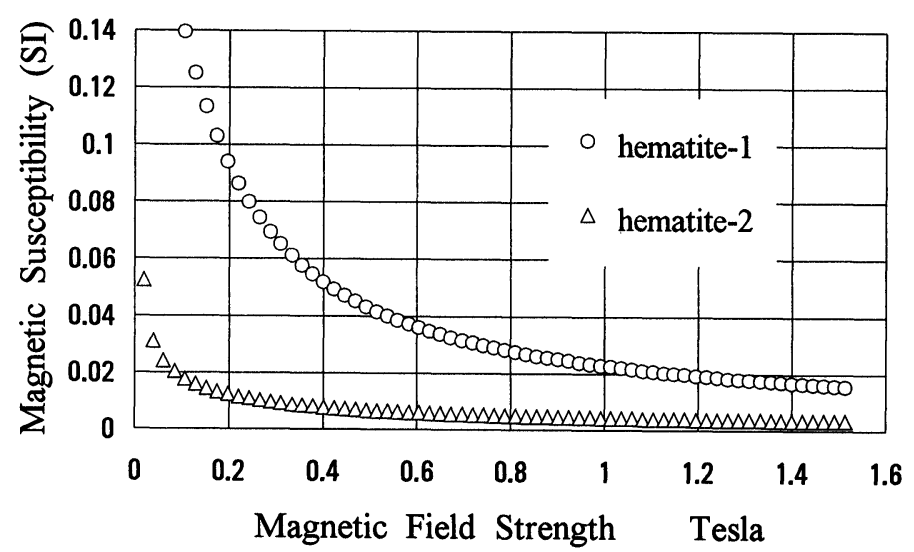

Fig. 8 Magnetic susceptibility curves of hematite minerals

It can be seen that the susceptibility of hematite-1 is nearly one order of magnitude higher than that of hematite-2. From eq. (3), this result seems to qualitatively explain why the flocculation efficiency of hematite-1 increased with 
the magnetic field strength and particle size, and was better than that of hematite-2. Also, without an applied magnetic field, owing to the remanent magnetisation of hematite-1 itself, a weak flocculation may occur, leading to the change of sediment percentage with pulp $\mathrm{pH}$.

It is worth noticing that hematite-1 is a strong paramagnetic mineral. Its magnetic behaviour is in some degree like a ferromagnetic material. When increasing the applied magnetic field strength from 0.1 to 1.0 Tesla, the susceptibility of hematite is reduced by an order of magnitude. This means that the magnetic interaction between hematite-1 particles does not increase obviously with the magnetic field. In this case, eq. (3) conventionally used cannot quantitatively explain the magnetic flocculation behaviour of hematite-1 particles, that is, for a given particle size, the magnetic interaction between hematite-1 particles is a constant and do not change with the applied magnetic field.

Svoboda [10] used this equation to calculate the threshold magnetic field for which the magnetic flocculation of siderite began. It was found that the calculated result was at least an order of magnitude lower than the experimental result. To explain why the flocculation of weakly magnetic mineral particles in a lower external magnetic field may occur, he developed a theoretical model of secondary minimum magnetic flocculation.

As can be seen from Figure 9, the value of total interaction between particles as a function of interparticle distance $r$ is characterised by several salient regions: primary minimum (PM), primary maximum ( $\mathrm{MX})$, and secondary minimum (SM). Their existence, depth (or height), and position are sensitive to change of $B$, $a, \psi_{0}, \kappa$ and $\chi$. By increasing $B$ for a given particle size $a$, SM may become deeper and its position shifts towards smaller $r$ values; simultaneously MX decreases and moves to higher $r$ values. The existence of SM implies that particles can flocculate into SM.

In this study, the flocculation behaviour of $-15+11 \mu \mathrm{m}$ hematite-2 particles may be explained by this model. When the magnetic field strength is between 0.3 and1.0 Tesla, the hematite-2 particles flocculate into SM, and the efficiency of magnetic flocculation was only improved very slightly with the increase of the field strength. 
when the field strength is greater than 1.0 Tesla, the particles flocculate into the primary minimum, and the flocculation efficiency increased with the field strength. Thus, the increase of flocculation efficiency of hematite-2 particles would be discontinuous with the increase of magnetic field strength. For hematite-1, owing to the larger value of magnetic susceptibility, it is possible that the position of secondary minimum shifts to such a degree that the position overlap of SM and PM has occurred. Therefore, the increase of flocculation efficiency would be continuous with an increase of magnetic field strength.

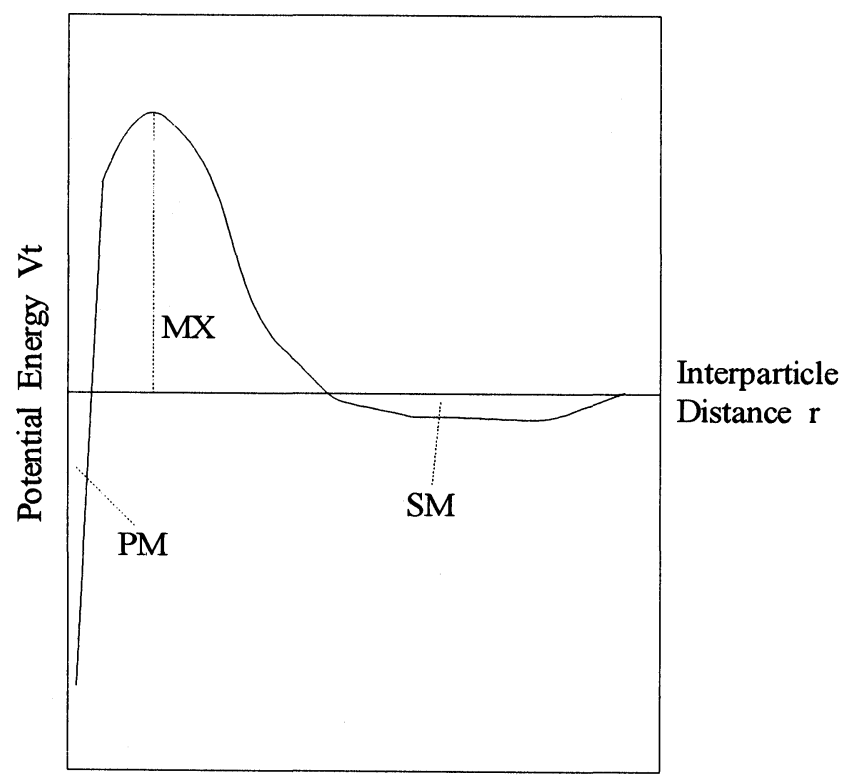

Fig. 9 The general form of the total potential energy for a pair of interacting colloidal particles

Conversely, in the process of flocculation, the formation of flocs commonly requires two preconditions, that is, the total interaction energy between particles is equal to or less than zero and there is enough probability of collision and attachment between particles. In a system of hydrophobic flocculation, the stirring speed and time are important variables [11]. The use of strong stirring is mainly to shorten the distance between particles colliding with each other to the situation of total interaction energy equal to zero. The use of long stirring times is mainly to increase the probability of collision between particles. 
However, the experimental results of magnetic flocculation indicated that only stirring of a certain time was required, while the stirring speed should be as slow as possible. It seems that in the process of magnetic flocculation, the effect of stirring speed is only to keep particles suspended. Thus, the behaviour of magnetic flocculation should be similar to coagulation using an electrolyte.

In fact, in the process of magnetic flocculation, the stirring not only increases the effect of shear force from the pulp, but also increases the effect of magnetic breakage force. When magnetic flocs are formed at one position within the magnetic field and move to a new position, it is very likely that their magnetisation direction is different from the applied magnetic field direction. In this case, the interaction between flocs magnetisation and applied magnetic field will cause the breakage of flocs. Therefore, magnetic flocculation requires stirring at a slow speed, and the rate of magnetic flocculation is slower than the one of common coagulation.

When a higher stirring speed is used, the collision probability between particles is increased so that the stirring time required can be shortened, but the flocculation efficiency is decreased because of the increase of shear force and magnetic breakage force with the stirring speed. When a higher magnetic field strength is used, the attractive interaction energy between particles is increased. The flocs formed will be able to withstand stronger breakage force. Thus, with the increase of magnetic field strength, the stirring time will be shortened and the flocculation efficiency will be increased.

\section{CONCLUSION}

It has been found that the magnetic flocculation of hematite-1 mineral may occur in a relatively weak applied magnetic field, and the flocculation efficiency increased with the magnetic field strength and particle size. The behaviour of magnetic flocculation of hematite-2 is more complicated, probably because of flocculation into the secondary minimum region of the total interaction energy to the distance between particles, in the range of magnetic field strength from 0.3 to 1.0 Tesla. 
Magnetic flocculation can be enhanced by stirring for a certain time, and increasing magnetic field strength may shorten the stirring time required. Magnetic flocculation requires slow speed stirring, because the stirring not only increases the effect of shear force from the pulp flow, but also increases the effect of magnetic breakage force.

It can also be found that the method used conventionally to calculate the magnetic interaction between particles is not suitable for the strongly paramagnetic mineral, hematite-1, because the magnetic susceptibility decreases with an increase of magnetic field strength.

\section{ACKNOWLEDGEMENTS}

The authors gratefully acknowledge the financial support provided by an ORS award and the Chemical Engineering School of the University of Birmingham.

\section{REFERENCES}

[1] D.F. Bagster and J.D. Mcilvenny: Studies in the flocculation of hematite from gangue using high molecular weight polymers. Int. J. Min. Proc. 14 $(1985), 1$

[2] R. Snamohan: The problem of recovering very fine particles in mineral processing: a review. Int. J. Min. Proc. 28 (1990), 247

[3] Y. Wang and E. Forssberg: Aggregation between magnetite and hematite ultrafines utilising remanent magnetisation. Miner. Eng. 5 (1992), 895

[4] Y. Wang, E. Forssberg and R.J. Pugh: Hydrophobic magnetite seeding of hematite ultrafines in high gradient magnetic separation. Miner. Eng. 6 (1993), 537

[5] Q.Y. Song, F. Xu and S.C. Tsai: Magnetic seeding flocculation of weakly magnetic iron minerals. Int. J. Min. Proc. 34 (1992), 219

[6] V. Hencl and J. Svoboda: The possibility of magnetic flocculation of weakly magnetic iron minerals. Proc. 13th Int. Min. Proc. Congress, Warsaw, Poland (1979), 209

[7] A. Cikcik and T.J. Veasey: Magnetic flocculation of weakly magnetic minerals. M. Phil. Thesis, University of Birmingham (1985) 
[8] R.J. Pugh, Y. Wang and E. Forssberg: The influence of magnetic and surface forces on the coagulation of hematite and chromite. Miner. Metall. Process. 11 (1994), 133

[9] J. Svoboda: A theoretical approach to the magnetic flocculation of weakly magnetic minerals. Int. J. Min. Proc. 8 (1981), 377

[10] J. Svoboda and J. Zofka: Magnetic flocculation in secondary minimum. $J$. Colloid Interface Sci. 94 (1982), 37

[11] L.J. Warren: shear-flocculation of ultrafine scheelite in scdium oleate solution. J. Colloid Interface Sci. 50 (1975), 307

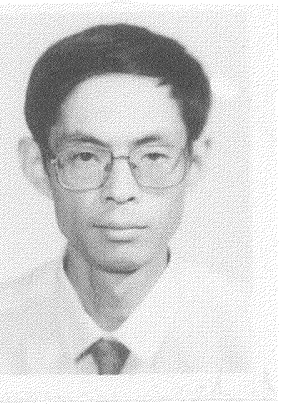

Yarui Shao received his B.Sc. in Mineral Engineering from the Northeast University in 1982 and obtained his M.Sc. in Mineral Engineering from the Beijing General Research Institute of Mining and Metallurgy (BGRIMM) in1985. After these, he joined the Mineral Engineering Department of the BGRIMM working on a wide range of research on mineral processing techniques. He is presently a Research Student for Ph.D. in the School of Chemical Engineering of the University of Birmingham.

N.A. Rowson: For biography see Magn. Elect. Sep. 3 (1992), 105

T.J. Veasey: For biography see Magn. Elect. Sep. 4 (1993), 148

Keywords: magnetic flocculation, hematite, recovery of fines 